EPiC Series in Engineering
Volume 3, 2018, Pages 535-540
HIC 2018. 13th International
Conference on Hydroinformatics

\title{
Building a nonlinear relationship between dew point temperature and precipitation to apply a method to downscale GCMs information: Case study in Santa Catarina River Basin, Monterrey
}

\author{
Del Castillo Castillo, J. L. ${ }^{13}$, Corzo Pérez, G. A. ${ }^{1}$, Bouwer, L. ${ }^{2}$, Ramírez \\ Orozco, A. I. ${ }^{3}$ and Bhardwaj, A. ${ }^{12}$ \\ 1IHE Institute for Water Education, Department P.O.Box 3015, 2601 DA, Delft, The Netherlands \\ 2 Deltares, PO Box 177, 2600 MH Delft, The Netherlands \\ 3 Tecnológico de Monterrey, Av. Eugenio Garza Sada Sur No. 2501, C.P. 64849 Monterrey, \\ Nuevo León, México \\ jkstillo85@gmail.com, g.corzo@un-ihe.org, \\ laurens.bouwer@deltares.nl, aldo.ramirez@itesm.mx, \\ ab.aashishbhardwaj@gmail.com
}

\begin{abstract}
.
This study will explore the use of a nonlinear relationship between dew point temperature (DPT) and precipitation to apply a method to downscale global circulation models (GCMs) information. Recently, there have been attempts to characterize dew point temperature (DPT) and precipitation intensity. The DPT has shown to be an easier variable to predict, and therefore forecast or prediction on GCM could provide a more reliable estimation. DPT using Clausius-Clapeyron (CC) relationship is one of the methods. This relationship has approximated a non-linear relation into a simple slope coefficient. In a recent study in the Netherlands, DPT was processed by the Advanced Delta Change Method (ADCM) and then converted to precipitation using CC or super $\mathrm{CC}$ relation observing better results. In this research work this approach is compared with a non-linear (neural network) model of the relationship. The main contribution of the overall project is to explore the improvement of a better relationship description and how much this could impact in our engineering intensity duration frequency curves. The problem posed is the analysis of the implications of climate change information for civil engineering design in the Metropolitan Area of Monterrey (Santa Catarina River Basin in Mexico).

Keywords: 3-6 keywords (in alphabetical order) Advanced Delta Change Method, Clausius-Clapeyron relationship, Climate Change, Dew point temperature, Downscaling, Neural network
\end{abstract}




\section{Introduction}

In the last decade, the scientific community have increasingly developed methods which predict behavior of more intense hydrologic phenomena. Projection of future precipitation extreme events have been the main focus of this new methodologies (Arnbjerg-Nielsen, 2012). Precipitation values from GCM present high variability and show to be one of the most complex variables in the prediction process. Recently, there have been attempts to characterize temperature and precipitation intensity, because temperature has shown to be an easier variable to predict. Variations in temperature have been used to analyze its relation to rainfall intensity. DPT using Clausius Clapeyron relationship (CC) is one of the methods to relate temperature and precipitation intensity. A 7\% increase in precipitation with an increase in DPT is the normal relation between those variables. Nevertheless, in recent studies driven by the Royal Netherlands Meteorological Institute (KNMI) they found a dependency that doubles the typical $7 \%$ CC relationship and they called it super CC (sCC) relation (Lenderink \& Attema, 2015) (Lenderink, Mok, Lee, \& Van Oldenborgh, 2011) (Lenderink \& van Meijgaard, 2010) (Lenderink \& van Meijgaard, 2008). The CC and $\mathrm{SCC}$ have shown to characterize well the relationship as well as to provide a basis for the analysis of future rainfall. In a master thesis conducted in Unesco-IHE together with KNMI and Deltares in 2017 in the Netherlands, dew point temperature was processed by the Advanced Delta Change Method (ADCM) and then converted to precipitation using a simple scale coefficient of the CC or sCC relation observing better results (Bhardwaj, 2017). The main objective of this research work is to develop a non-linear model (neural network) to have a better approximation of the CC relationship. In order to fulfil the previous goal, IDT(F) curves where used to test and compare this new approach with the previous methodologies in the Santa Catarina River Basin, Monterrey.

\section{Material and Methods}

The GCM selected to this study was the CMIP5 multi-model ensemble IPSL-CM5A-MR from the Institute Pierre-Simon Laplace for a radiative forcing pathway RCP4.5 experiment (World Climate Research Programme, 2012). ERA-INERIM reanalysis was the database selected as the observation dataset and it was corrected using the information of the climatology stations available in the zone (Dee, et al., 2011). The control and observation period selected was 1981-2010, and two future periods were selected 2041-2070 and 2071-2100.

The Santa Catarina River Basin has a total area of $1807 \mathrm{~km}^{2}$ and was selected to analyze the implications of the novel methodology in climate change. In Figure 1 is shown that the Metropolitan Area of Monterrey (MAM) is located in this basin, in the northeast part of Mexico and it is the third biggest urban center of the country (Tecnológico de Monterrey \& CONAGUA, 2010). MAM is formed by thirteen municipalities and has a population of more than four million inhabitants according to the projections of Consejo Nacional de Población (CONAPO) for 2014. According to Comisión Nacional del Agua (CONAGUA) four or five severe storms occur in the MAM each year. 


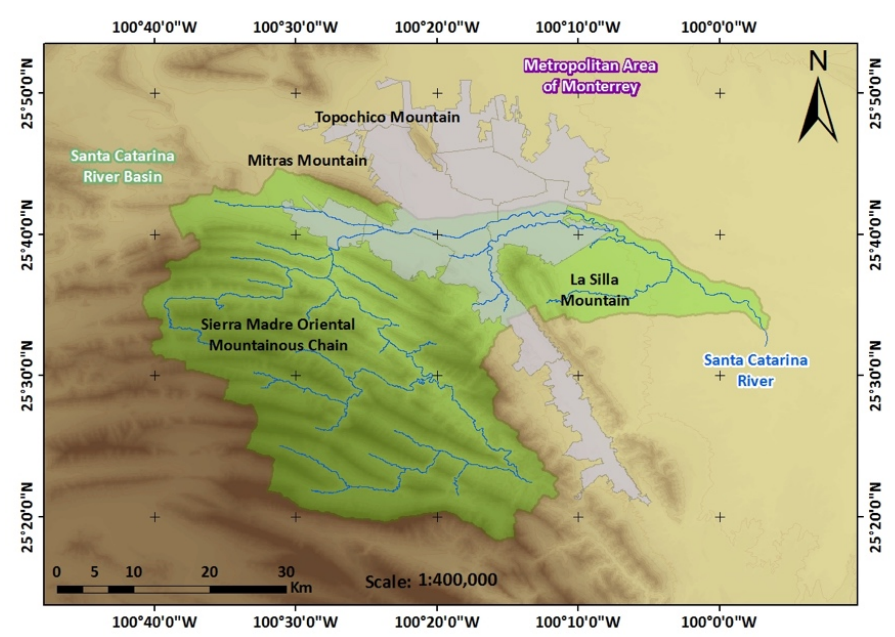

Figure 1: Santa Catarina River Basin (Own Author)

\subsection{Methodology}

Three methodologies are performed and compared. In the first one, ADCM is used to transform observed precipitation into future precipitation through the meteorological data projected in the GCMs. (Kraaijenbrink, 2013). In the second one, ADCM transformed observed DPT into future DPT and then, through an exponential equation convert observed precipitation into future precipitation using a slope coefficient (ADCM-DPT). The slope coefficient is defined as the percentage of increase in observed precipitation with a degree increase in DPT. A critical point is defined to identify two relationships, $7 \%$ (CC) and 14\% (sCC). The difference between media future DPT and media observed DPT is used as exponential coefficient on the equation 1 (Bhardwaj, 2017). In the third one, a non-linear model is used instead of a linear approximation to reproduce the results of the ADCM-DPT methodology. In this case, as first step in the development of a neural network, multilayer perceptron tool of WEKA software is used to see if a neural network is capable of reproduce the results of the ADCM-DPT procedure. An autocorrelation analysis is driven to know the lag period to use as input. The general procedure following in this project is shown in Figure 2.

$$
P^{*}=P_{o} \times\left(1+C C_{r}\right)^{\left(\overline{D P T_{f}}-\overline{D P T_{o}}\right)}
$$

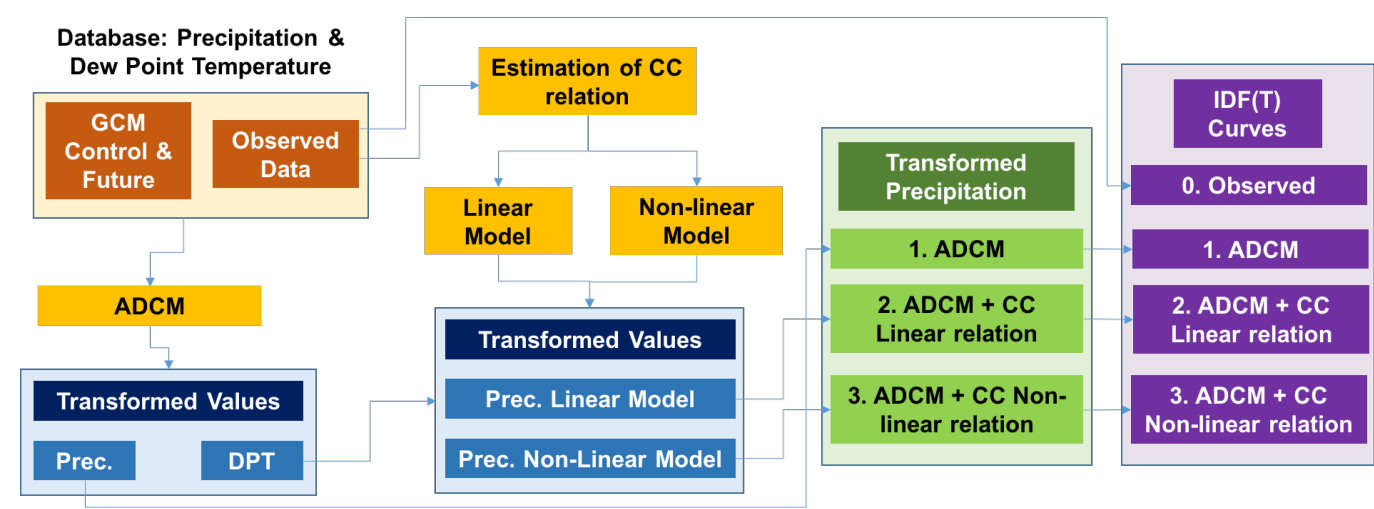

Figure 2: General scheme of the methodology following to realize this research work. 


\section{Results and Discussion}

In the linear model, the CC relation was performed for six different percentiles $(99.9,99,95,90,80$ and $70 \%$ ). Figure 3 shows how the non-linear behavior of the relationship between dew point temperature and precipitation can adjust to a $7 \%$ (green slashed line) or 14\% (red slashed line). Also, it can be seen that the relationship is not always 7 or 14 percentage. In that case, a neural network approximation is a better representation of the relation. The comparison between the IDF curves of the observed, ADCM and ADCM-DPT, shows that for the case of the Santa Catarina River Basin, the ADCM shows the most extreme behavior as it can be seen in Figure 4.
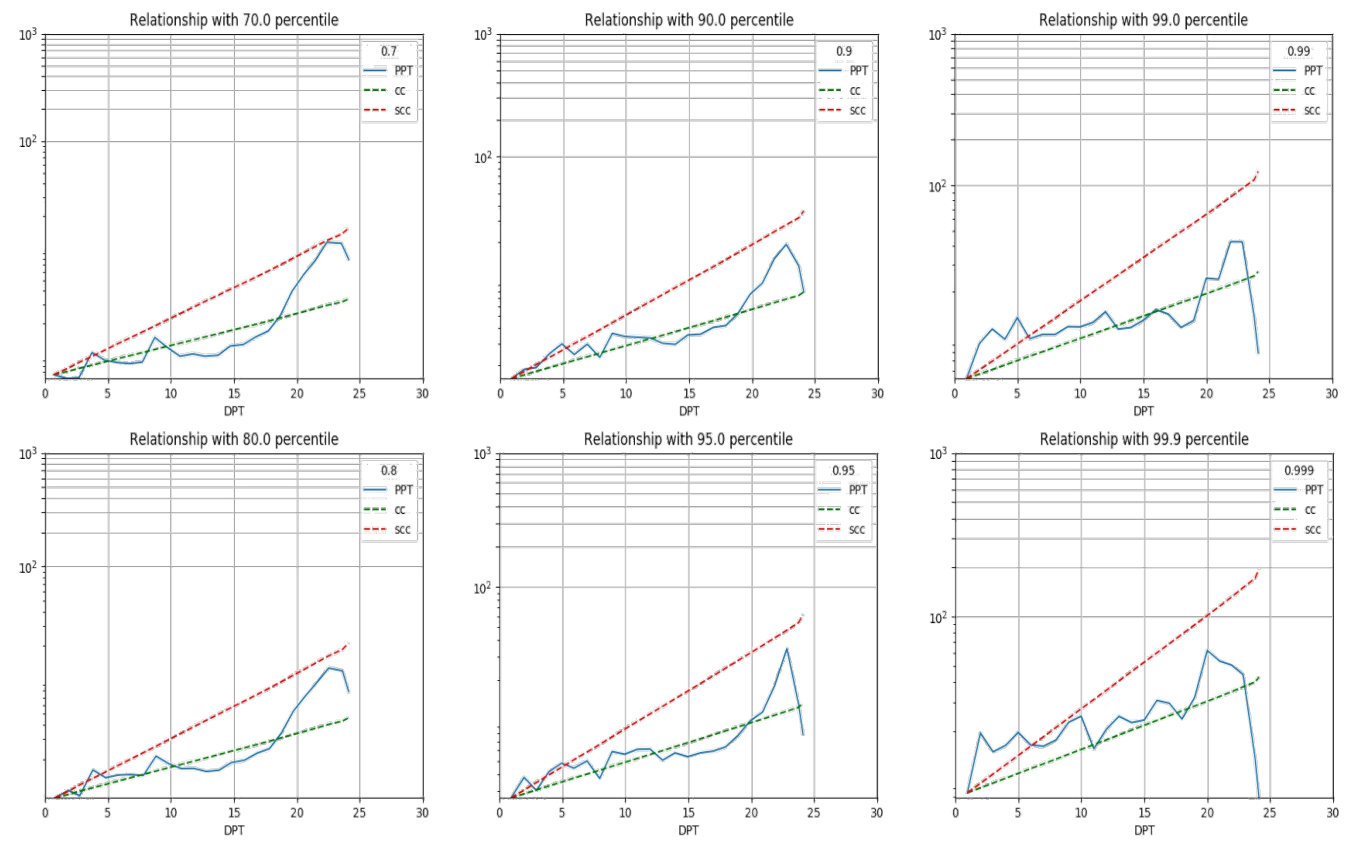

Figure 3: Dew point temperature versus precipitation intensity obtained for 70, 80, 90, 95, 99, and 99.9 percentile in the Santa Catarina River Basin.
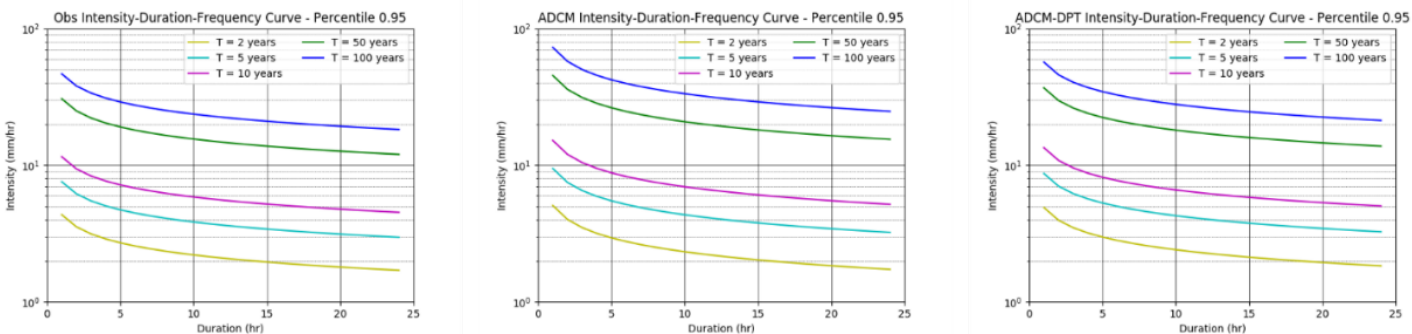

Figure 4: IDF curves for a 95 percentile. 
The first attempt to adjust an artificial neural network consisted on use a data driven model to predict ADCM-DPT future precipitation to the dew point temperature - precipitation phenomena. The point of that is to see if the ANN is capable to reproduce the future DPT results. In order to achieve that the observation values and the observation values (DPT and precipitation) with a lag are entered as an input in the ANN, as an output value the results of the ADCM-DPT precipitation values are used. WEKA software is used as the computational tool to apply the ANN. An autocorrelation is driven in order to calculate the lag of the observed precipitation to introduce in the model. In Figure 5 can be seen that the value of the lag that is used over the precipitation data is six.

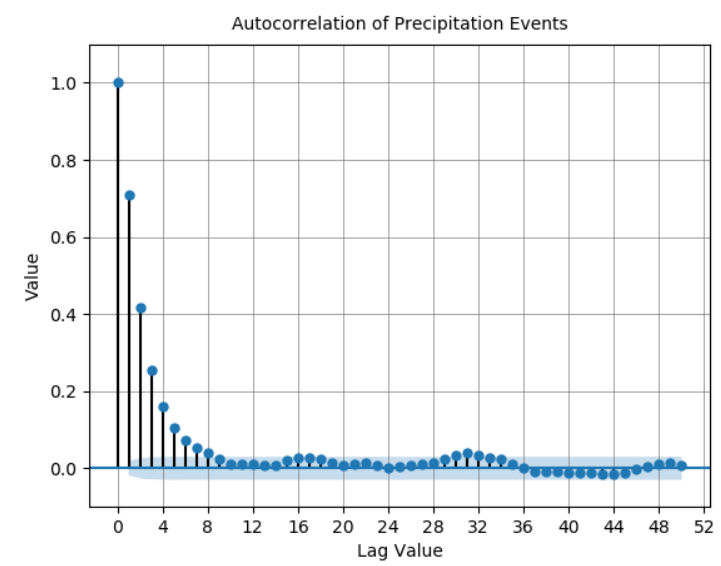

Figure 5: Autocorrelation of the precipitation events

The predicted result from the ANN had a 0.98 correlation between the expected precipitation result from the ADCM-DPT. As can be seen in Figure 6 both results are very similar.

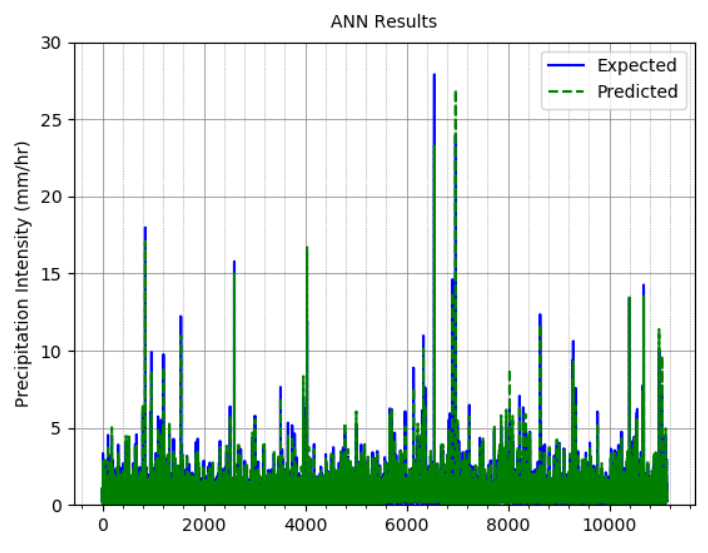

Figure 6: Expected precipitation calculated by ADCM-DPT vs. predicted precipitation. 


\section{Conclusions}

The results of the ADCM shows the most extreme conditions, which contradicts the results found by KNMI, where ADCM-DPT is the worst scenario. The behavior of the CC relationship depends on the region of study. In the Santa Catarina River basin, the percentage of increase between the DPT and precipitation tends to be more than a super CC relation (14\%) after reaching the critical point but it looks like to adjust very well to a CC relation for temperatures below the $18^{\circ} \mathrm{C}$. In order to get a better $\mathrm{CC}$ relation to convert dew point temperature to precipitation a data driven model to describe the phenomena was developed. The adjusted neural network can reproduce the results of the ADCM-DPT procedures. But, improvements in intermediate steps must be done in order to develop a more accurate procedure.

\section{References}

Arnbjerg-Nielsen, K. (2012). Quantification of climate change effects on extreme precipitation used for high resolution hydrologic design. Urban Water Journal, 57-65.

Bhardwaj, A. (2017). Future precipitation extremes: Comparing GCM downscaling and temperature scaling methods for urban flood hazards.

Dee, D., Uppala, S., Simmons, A., Berrisford, P., Poli, P., Kobayashi, S., . . Vitart, F. (2011). The ERA-Interim reanalysis: Configuration and performance of the data assimilation system. Quarterly Journal of the Royal Meteorological Society, 137(656), 553-597.

Kraaijenbrink, P. (2013). Advanced Delta Change method. De Bilt.

Lenderink, G., \& Attema, J. (2015). A simple scaling approach to produce climate scenarios of local precipitation extremes for the Netherlands. Environmental Research Letters, 10(8), 85001.

Lenderink, G., \& van Meijgaard, E. (2008). Increase in hourly precipitation /'extremes beyond expectations from temperature changes. Nature Geoscience, 1(8), 511-514.

Lenderink, G., \& van Meijgaard, E. (2010). Linking increases in hourly precipitation extremes to atmospheric temperature and moisture changes. Environmental Research Letters, 5(2), 25208.

Lenderink, G., Mok, H., Lee, T., \& Van Oldenborgh, G. (2011). caling and trends of hourly precipitation extremes in two different climate zones - Hong Kong and the Netherlands. Hydrology and Earth System Sciences, 15(9), 3033-3041.

Tecnológico de Monterrey, \& CONAGUA. (2010). Estudio Hidrológico de la Cuenca del Río Santa Catarina, N.L. Monterrey. Monterrey.

World Climate Research Programme. (2012). Coupled Model Intercomparison Project Phase 5 Data Archive. Retrieved September 2017, from http://cmip-pcmdi.llnl.gov/index.html 